\title{
Electrobioremediation of an unsaturated soil contaminated with hydrocarbon after landfarming treatment
}

\author{
A.J. Acuña, ${ }^{1, *}$ N. Tonin, ${ }^{1}$ G.N. Pucci, ${ }^{1}$ L. Wick, ${ }^{2}$ O.H. Pucci ${ }^{1}$ \\ ${ }^{1}$ Centro de Estudios e Investigación en Microbiología Aplicada, Universidad Nacional de la \\ Patagonia San Juan Bosco, Apartado postal 9000, Ciudad Universitaria, \\ Comodoro Rivadavia, Argentina \\ ${ }^{2}$ UFZ, Centro de Investigaciones Ambientales Leipzig-Halle. Departamento de Microbiología \\ Ambiental. Permoserstrasse 15, Leipzig, Alemania
}

Received 17 November 2009; accepted 15 September 2010

\begin{abstract}
The electro-bioremediation is a technique that is used for the remediation of hydrocarbon contaminated soils. The aim of this study is to explore the electrobioremediation of an unsaturated soil, contaminated with hydrocarbon waste generated by the oil industry activity in the area and previously remediated by landfarming, order to increase the removal of polyaromatic hydrocarbons.

The sample was put in a three-compartment electro-bioremediation glass cell of $58 \mathrm{~cm}$ long, the lateral compartments containing the electrolyte; we used bridges of ammonium phosphate to connect the electrolyte with the soil sample in the central compartment. A potential difference of $0.5 \mathrm{~V} \mathrm{~cm}^{-1}$ was applied to the electrobioremediation cells for 60 days. A second cell was used for control and no current was applied to it. The monitoring was carried out by a counting cell and measuring of nalkanes and polyaromatic hydrocarbons using GC mass. The results showed that this technology has good potential to increase the biodegradation of n-alkane hydrocarbons and polyaromatic hydrocarbons such as phenanthrene, 1-3-metilphenanthrene, chrysene, 3-methylchrysene, 6-methylchrysene, benzo(b)fluoranthrene and benzo(ghi)pyrene which, without the application of direct current, were not biodegraded by microorganisms in the soil. The use of salt bridges maintained the $\mathrm{pH}$ between values that are compatible with the degrading bacterial community.
\end{abstract}

Keywords: electrobioremediation, $\mathrm{PAH}$, bacteria, unsaturated soil.

\footnotetext{
* Corresponding author. E-mail address: ajcuna@unpata.edu.ar
} 


\section{Introducción}

En la región del Golfo San Jorge, se lleva a cabo una gran actividad de la industria dedicada a la extracción de hidrocarburos. Esta actividad se realiza desde hace más de cien años y, durante este período, se fueron modificando las legislaciones ambientales nacionales y provinciales, haciéndose más exigentes para lograr una mejor calidad de los ambientes contaminados con hidrocarburos. El tratamiento de los suelos contaminados llevó a desarrollar diferentes técnicas para la eliminación de los hidrocarburos que poseen los suelos de la Patagonia. Muchos de ellos tienen años de contaminación por hidrocarburos sin ningún tipo de asistencia y control. En la actualidad, en la región se utilizan las técnicas de landfarming, biopilas y biorremediación asistida in situ para resolver estos problemas de contaminación. Estos dan excelentes resultados cuando se aplican con rigurosidad y un monitoreo constante [1]. Los hidrocarburos alifáticos se eliminan con mayor velocidad, mientras que más lentamente son eliminados los hidrocarburos aromáticos y polares [2]. Uno de los inconvenientes de estos últimos, es la baja biodisponibilidad que poseen [3], lo que se ha tratado de resolver con el agregado de surfactantes, que en la mayoría de los casos pueden producir lixiviados importantes.

Hay numerosos estudios sobre biodegradación de hidrocarburos que demuestran la importancia y aplicabilidad de la técnica con cualquiera de sus variaciones, biorremediación in situ, landfarming, biopilas, etc., en suelos patagónicos [2, 4, 5]. En estos estudios, en general se llega a la conclusión que es una tecnología aplicable en base a su ecuación costo beneficio conveniente [6]. En el caso de landfarming se detectó que, si bien los valores de hidrocarburos totales suelen ser menores a los permitidos por la legislación regional vigente, cuando se estudiaban individualmente los hidrocarburos poliaromáticos (PAH), no siempre cumplían con la misma.

La electrorremediación es una tecnología para restaurar suelos contaminados por la acción de un campo eléctrico a partir de la aplicación de corriente directa [7]. Los mecanismos de remoción principales son la electromigración, la electroósmosis, y la electroforesis [8]. La electroósmosis se produce en función del grado de saturación del suelo, por ello es un factor primordial en la electrobiorremediación (EBR). Se observa una distribución no homogénea de la humedad del suelo ya que el agua se mueve en dirección de uno de los electrodos, el cátodo, aumentando la humedad de este y disminuyendo en la región del ánodo. Esta disminución se encuentra asociada con el desarrollo de poros con presión negativa, variación del $\mathrm{pH}$ y de la fuerza del campo eléctrico, lo cual resulta en una tasa de flujo electroosmótico irregular [9]. Se ha demostrado su capacidad para remover algunos contaminantes orgánicos como tricloroetileno e hidrocarburos en estudios de laboratorio. La aplicación que se ha comunicado con más frecuencia es la de recuperación de suelos contaminados con metales pesados como $\mathrm{Cr}, \mathrm{Cd}, \mathrm{Hg}, \mathrm{Pb}, \mathrm{Mn}, \mathrm{Zn}$ y As $[10,11,12,13,14]$.

La EBR combina la aplicación de corriente eléctrica directa con técnicas de biorremediación asistida con nutrientes, aireación y agua. Se busca estimular la biodegradación de contaminantes orgánicos al introducir nutrientes para los microorganismos dentro del suelo, de manera que el campo eléctrico acelere la 
desorción de los contaminantes desde las partículas de suelo, incrementando su biodisponibilidad para que los microorganismos los incorporen a su metabolismo [15]. Esta modificación ha sido probada con moléculas orgánicas sintéticas como TNT, BPC y pesticidas [16], siendo la humedad, el pH, la naturaleza química del contaminante y las características y el potencial zeta $(\zeta)$ del suelo, los factores más relevantes que influyen en el proceso [17].

Este trabajo tuvo como objetivo estudiar electrobiorremediación en un suelo no saturado con una contaminación por hidrocarburos de más de cinco años, para aumentar la eliminación de los hidrocarburos poliaromáticos existentes en la muestra.

\section{Materiales y métodos}

\section{Muestra de suelo}

Se trabajó con suelo proveniente de un sistema de biorremediación por el método de landfarming ubicado al norte de la ciudad de Comodoro Rivadavia. Se colectaron 20 muestras, durante la primavera, a una profundidad comprendida entre 10 y $30 \mathrm{~cm}$ que se mezclaron y se redujeron por cuartetos. El peso final de muestra fue de $10 \mathrm{~kg}$.

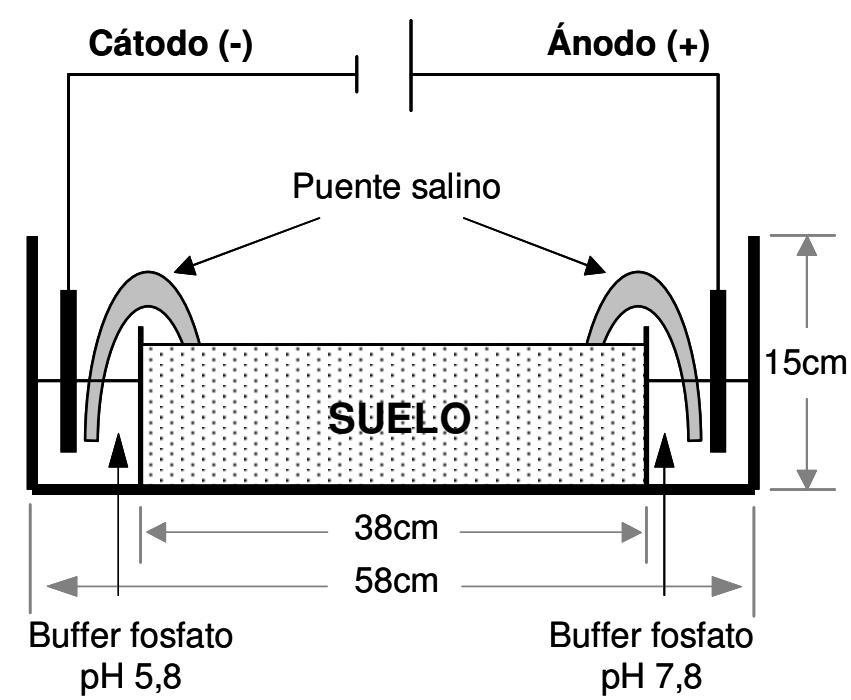

Figura 1. Esquema de la celda electroquímica utilizada en la experiencia de electrobiorremediación.

\section{Experiencia de electrobiorremediación}

Las dimensiones de la celda de vidrio utilizada fueron de $58 \mathrm{~cm}$ de largo, $15 \mathrm{~cm}$ de alto, $15 \mathrm{~cm}$ de ancho. El interior de esta estuvo dividido en tres compartimientos, dos de ellos de $10 \mathrm{~cm}$ de largo, $15 \mathrm{~cm}$ de alto y $15 \mathrm{~cm}$ de ancho, ubicados uno a cada extremo de las cubas donde se colocaron las soluciones buffer. El tercer compartimiento fue el central, de $38 \mathrm{~cm}$ de largo, $15 \mathrm{~cm}$ de alto y $15 \mathrm{~cm}$ de ancho, donde se colocó el suelo para las experiencias de EBR (Fig. 1). Se utilizó una solución de buffer $1 \mathrm{M}\left(\mathrm{K}_{2} \mathrm{HPO}_{4} / \mathrm{KH}_{2} \mathrm{PO}_{4}\right)$ ajustado a pH 7.8 para el compartimiento del ánodo y a pH 5.8 para el del cátodo [18]. En estas soluciones fueron sumergidos los electrodos de platino y el paso de corriente a través del suelo se logró por la unión de éste, con las soluciones buffer, utilizando 
puentes salinos de fosfato de amonio. La incubación se realizó a $24{ }^{\circ} \mathrm{C}$ durante 60 días. El voltaje aplicado al suelo fue de $0.5 \mathrm{Vcm}^{-1}$ con una humedad entre 12-15\%. Como control se utilizó un sistema de iguales características a lo mencionado anteriormente, sin la aplicación de corriente directa. Al inicio y al final de la experiencia se realizaron análisis físicos, químicos, bacteriológicos y de hidrocarburos. Para esto se tomaron $15 \mathrm{~g}$ de suelo en forma estéril en las diferentes regiones estudiadas, cátodo, centro y ánodo, de los que un gramo se utilizó para los estudios microbiológicos, cinco gramos para los análisis de hidrocarburos y el resto para los estudios físicos y químicos.

\section{Análisis físico y químico}

Las determinaciones de $\mathrm{pH}$, cationes y aniones se realizaron sobre un extracto de suelo 1:2.5 en agua destilada. La determinación de $\mathrm{pH}$ fue realizada con electrodo de vidrio. El contenido de amonio como azul de indofenol y el de fosfato con azul de molibdeno. El calcio se determinó por complejometría con EDTA a pH 12, utilizando murexida como indicador y el nitrato con brucina en presencia de ácido sulfúrico [4]. Las determinaciones de temperatura y humedad se realizaron según lo propuesto por García Trejo en 1981 [19]. Los hidrocarburos n-alcanos y PAH fueron determinados por cromatografía gaseosa acoplada a masa según lo propuesto por el método 8015 de EPA [20]. El potencial zeta del suelo se determinó solo en la muestra inicial sobre $1 \mathrm{~g}$ en un equipo Zeta master Malvern Instrument Ltd malvern Worcestershire UK a 100 volt según Wick et al. [21].

\section{Análisis bacteriológico}

El número de microorganismos heterótrofos y de bacterias degradadoras de hidrocarburos (BDH) se realizó por recuento en placa en los medios R2A [22] y MM-PGO [5], respectivamente. La incubación de las placas se realizó en oscuridad durante 20 días a $28{ }^{\circ} \mathrm{C}$.

\section{Resultado y discusión}

El suelo presentó un potencial zeta de -25.30 , indicando que la carga neta de sus partículas es negativa. Esto generará que la electroósmosis en el suelo siga una dirección desde al ánodo hacia el cátodo. Los valores del análisis químico realizado (Tabla 1) fueron congruentes con lo observado en suelos patagónicos estudiados anteriormente [4, 23, 24].

La función del amonio y del nitrato en los sistemas de biorremediación es de nutriente. La adición de ambos en estos sistemas es para otorgar al suelo cantidades necesarias de nitrógeno. El amonio en sistemas de EBR con suelos saturados disminuye su concentración debido a que, posiblemente, se transforma a amoníaco gaseoso [25]. En el sistema sometido al campo eléctrico, se detectó olor a amoníaco en los 30 días finales del ensayo sugiriendo la reducción del amonio a amoníaco gaseoso. En suelos saturados, también se observó que el fosfato disminuye su concentración en la región del cátodo, pero éste no es transportado hacia el ánodo debido a la presencia de calcio, ya que precipita en combinaciones de fosfato y carbonato de calcio [25]. En el presente trabajo, la concentración de fosfato en el suelo no aumentó, sino que disminuyó, 
probablemente por su utilización por las bacterias del suelo al biodegradar los hidrocarburos. La presencia de calcio es otro de los motivos por los que pudo disminuir su concentración, por las mismas razones determinadas por Schmidt et al. en 2007 [25], es decir, que ambos iones precipiten en forma de fosfato de calcio (Tabla 1).

Tabla 1. Análisis químico del suelo en el inicio y al final de la experiencia. Los valores del los aniones y cationes se expresan en $\mathrm{mg} \cdot \mathrm{kg}^{-1}$. El número de microorganismos se expresa como UFC.g ${ }^{-1}$ de suelo. $\mathrm{BH}$ : bacterias heterótrofas, BDH: bacterias degradadoras de hidrocarburos.

\begin{tabular}{lccccc}
\hline Diadema & Inicial & Control & Cátodo & Centro & Ánodo \\
\hline Fosfato & 32 & 29 & 12 & 20 & 27 \\
Calcio & 75.5 & 81.0 & 108.2 & 81.0 & 103.2 \\
Nitrato & 17.4 & 18.9 & 29.8 & 28.9 & 36.3 \\
Amonio & 0.8 & 0.8 & 0.8 & 1.1 & 80.3 \\
BH & $3.1 \times 10^{7}$ & $2.4 \times 10^{7}$ & $1.0 \times 10^{8}$ & $1.6 \times 10^{8}$ & $2.7 \times 10^{7}$ \\
BDH & $1.4 \times 10^{8}$ & $8.1 \times 10^{6}$ & $1.1 \times 10^{7}$ & $1.8 \times 10^{7}$ & $1.9 \times 10^{7}$ \\
\hline
\end{tabular}

La electroósmosis en un suelo se encuentra en función del grado de saturación, por ello es un factor primordial en la EBR [26]. Los sistemas saturados tienen el problema de mantener constante la humedad, y la circulación del agua también elimina hidrocarburos obteniéndose un volumen de líquido contaminado que posteriormente debe ser remediado. Una de las ventajas de trabajar en sistemas no saturados, es que no se producen lixiviados, aunque el mantenimiento de la humedad de las cubas conlleva a un trabajo de seguimiento semanal para mantener el valor de la misma entre $12-15 \%$, ya que la caída de los valores de humedad implican un aumento del voltaje que se debe aplicar a los electrodos para mantener su valor constante en el suelo. Esto lleva a un mayor gasto de energía y menor rendimiento del sistema. La disminución de la humedad del sistema de EBR también pudo deberse a factores de calentamiento o al desarrollo de reacciones químicas exotérmicas [27]. La temperatura del suelo se mantuvo en $24.3 \pm 1{ }^{\circ} \mathrm{C}$ durante todo el ensayo, observándose una diferencia de $0.4{ }^{\circ} \mathrm{C}$ entre la región del ánodo y la del cátodo, con una temperatura ambiental de $24 \pm$ $2{ }^{\circ} \mathrm{C}$ y una temperatura en el suelo del control de $23,2 \pm 1{ }^{\circ} \mathrm{C}$. Esto indica que los cambios en la humedad en el sistema de EBR se debieron principalmente al flujo electroosmótico, concordando esto con otros autores que trabajaron con un voltaje de $0.3 \mathrm{Vcm}^{-1}[25]$.

Harbottle et al. [28] concluyó que la aplicación de un campo eléctrico en suelos no saturados produce mayores cambios en sus propiedades, produciendo un mayor impacto sobre la actividad microbiana y la biodegradación. Los puentes salinos mantuvieron el $\mathrm{pH}$ de suelo en forma aceptable (Fig. 2) durante los primeros 30 días. En los segundos 30 días, se observó un aumento de pH en las tres regiones estudiadas en la experiencia de EBR. Este aumento pudo estar asociado al ingreso de amonio al suelo, desde el puente salino, en la región del ánodo y su posterior transformación a gas amoníaco por efecto de la corriente aplicada. Sin embargo, este aumento de $\mathrm{pH}$ no provocó modificaciones en el número de $\mathrm{BDH}$ responsables de metabolizar el hidrocarburo contaminante 
(Tabla 1). El número de bacterias heterótrofas y de BDH se corresponde con los valores obtenidos para suelos patagónicos estudiados anteriormente por este grupo. El voltaje y las modificaciones de $\mathrm{pH}$ no presentaron impacto en los recuentos bacterianos manteniéndose en los valores iniciales y en un valor aceptable para la degradación bacteriana de hidrocarburos [2, 4, 23]. Los cambios de $\mathrm{pH}$ de los suelos generan carga en la membrana de las bacterias que, de esta manera, migran hacia uno de los electrodos dependiendo del valor de $\mathrm{pH}$ [29]. A pH 7 o mayor, se genera una carga negativa en la membrana que produce una migración de los microorganismos en dirección al ánodo [30], si bien la velocidad en medios acuosos es rápida, $5 \mathrm{cmh}^{-1}$, en suelos es menor, cayendo este valor a $5 \mathrm{~cm}$ cada $6 \mathrm{~h}$. Cuando el $\mathrm{pH}$ es de 5.5 o menor, se modifica la carga de la membrana siendo esta positiva, produciéndose un cambio de dirección hacia el cátodo. La complejidad de las cargas superficiales que poseen las membranas bacterianas hace difícil poder predecir el comportamiento de las mismas con los parámetros biofísicos [31]. Esto se explica porque la composición de los polímeros de las bacterias contiene grupos carboxílicos, sulfatos, fosfatos o aminos, por lo que posee grupos ácidos y básicos dando una superficie anfotérica que posee carga negativa a $\mathrm{pH}$ alto y carga positiva a $\mathrm{pH}$ bajo.

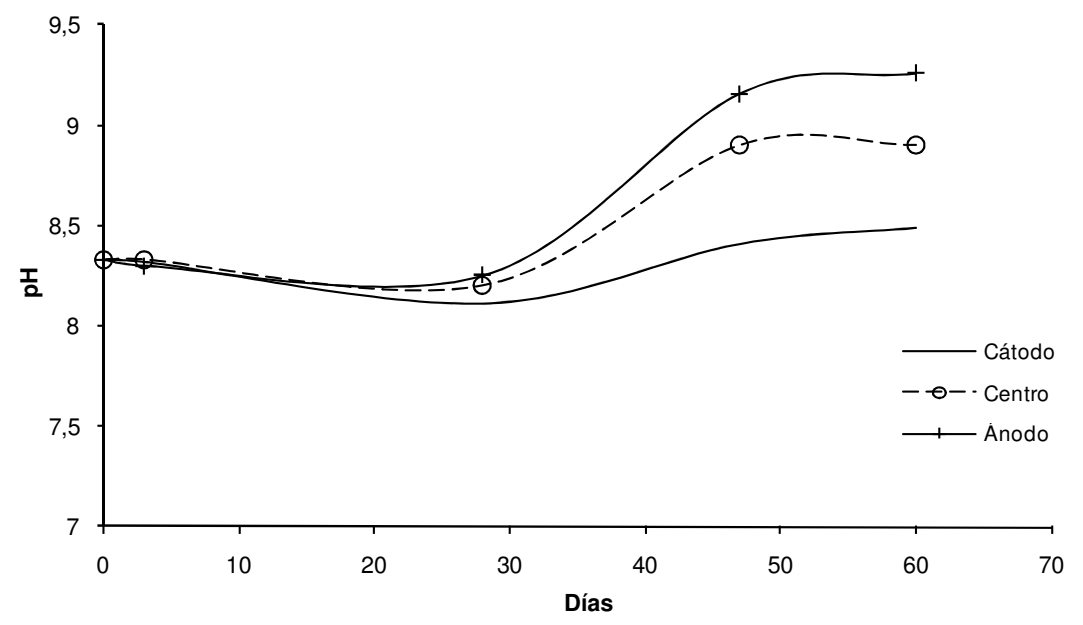

Figura 2. Seguimiento del $\mathrm{pH}$ en los diferentes sectores del sistema de elctrobiorremediación diseñado.

La aplicación del campo eléctrico en sistemas saturados acelera la biodegradación de PAH como el fenantreno, debido a la movilización de los fluidos asociados a la electroósmosis [18]. La mayor disminución de los hidrocarburos alifáticos se observó en el suelo cercano al ánodo, mientras que la mayor disminución de PAH se observó en la región cercana al cátodo (Fig. 3). 


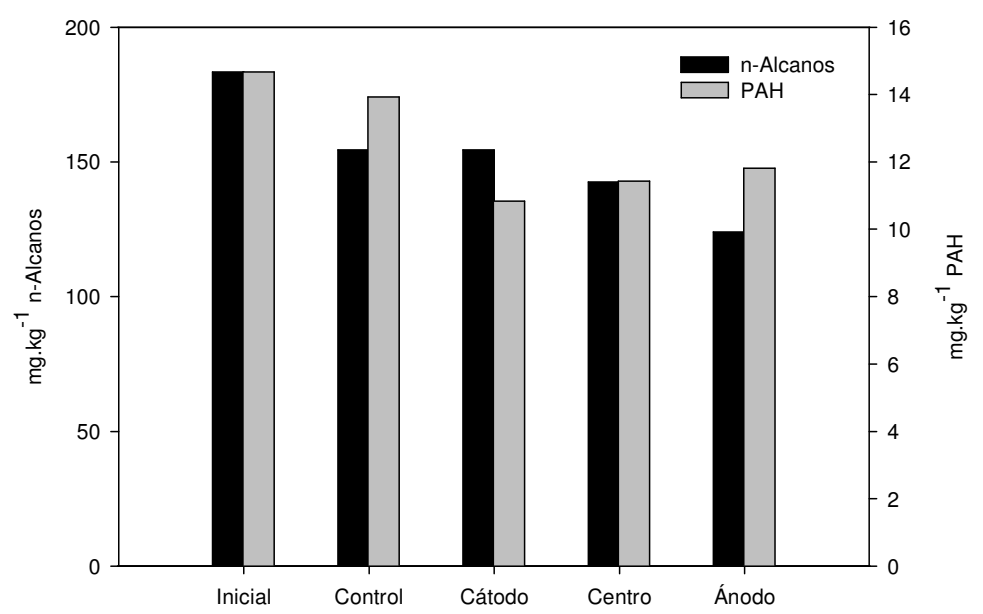

Figura 3. Hidrocarburos n-alcanos y poliaromáticos totales al inicio y final de la experiencia.

Con respecto a los hidrocarburos n-alcanos analizados, se observó que en el sistema utilizado como control hubo un porcentaje de biodegradación de $16 \%$. En el sistema de EBR, el porcentaje de biodegradación fue de 16\%, 22\% y 32\% para la región del cátodo, del centro y del ánodo, respectivamente, siendo en el suelo cercano a la región del ánodo, el sector donde se observó la máxima capacidad de biodegradación de n-alcanos. En el sistema control, los n-alcanos más utilizados fueron el triacontano en $47 \%$ y el hexacosano en $42 \%$. El heptadecano y dotriacontano fueron biodegradados en $38 \%$ y el octadecano en $37 \%$. Los n-alcanos eicosano y docosano fueron biodegradados en $34 \%$ y $19 \%$, respectivamente (Tabla 2 ).

Tabla 2. Concentración de los hidrocarburos n-alcanos determinados al inicio y al final de la experiencia. Los valores se encuentran expresados en mg. $\mathrm{kg}^{-1}$.

\begin{tabular}{lccccc}
\hline \multicolumn{1}{c}{ n-Alcano } & Inicial & Control & Cátodo & Centro & Ánodo \\
\hline Decano (C10) & 4.13 & 3.87 & 3.26 & 3.48 & 3.34 \\
Dodecano (C12) & 6.85 & 6.32 & 5.03 & 6.27 & 5.78 \\
Tetradecano (C14) & 19.75 & 20.80 & 18.09 & 16.67 & 21.30 \\
Hexadecano (C16) & 25.58 & 23.84 & 19.76 & 11.92 & 9.13 \\
Heptadecano (C17) & 2.68 & 1.67 & 2.50 & 2.68 & 2.24 \\
Octadecado (C18) & 10.51 & 6.57 & 10.42 & 10.10 & 7.57 \\
Eicosano (C20) & 14.25 & 9.36 & 20.20 & 16.64 & 12.47 \\
Docosano (C22) & 12.81 & 10.34 & 13.85 & 13.95 & 10.06 \\
Tricosano (C23) & 4.48 & 6.00 & 3.27 & 4.22 & 2.65 \\
Hexacosano (C26) & 25.50 & 14.82 & 16.51 & 17.99 & 10.89 \\
Octacosano (C28) & 12.54 & 17.86 & 12.81 & 8.18 & 8.81 \\
Triacontano (C30) & 14.10 & 7.43 & 5.82 & 6.95 & 4.78 \\
Dotriacontano (C32) & 8.41 & 5.18 & 6.26 & 5.73 & 7.08 \\
n-Alcanos totales & 183.46 & 154.48 & 154.53 & 142.56 & 124.03 \\
\hline
\end{tabular}

Por otro lado, en el sistema de EBR se observó que fueron biodegradados los mismos n-alcanos que en el sistema control, con la excepción de que en el suelo de la región del cátodo y del centro, ya que ahí no fueron utilizados los n-alcanos 
de entre 17 y 22 átomos de carbono (Tabla 2). En este sistema, la aplicación de corriente directa provocó la biodegradación del decano, dodecano, hexadecano, tricosano y octacosano. Sin embargo, los hidrocarburos n-alcanos no son un problema en los sistemas de biodegradación por técnicas convencionales en los suelos patagónicos [5, 23].

Los PAH suelen representar un problema en los sistemas de biorremediación. Se observó un porcentaje de biodegradación de aproximadamente $5 \%$ en el sistema utilizado como control mientras que en el sometido a corriente directa, los PAH fueron biodegradados en $26 \%, 22 \%$ y $19 \%$ en la región del cátodo, del centro y del ánodo, respectivamente (Fig. 3). El campo eléctrico utilizado fue capaz de aumentar la biodisponibilidad de los PAH [21] y de esta forma aumentó su biodegradación debido a que se metabolizan por oxidación. La aplicación de corriente directa aumentó de forma significativa estos valores, siendo mayor su efecto en el suelo cercano al cátodo. En el sistema control, los PAH biodegradados en mayor medida fueron el 1,7 dimetilfenantreno en un $22 \%$, el 1,6 dimetilnaftaleno y acenaftileno, ambos en un 17\%. El naftaleno, pireno y benzoantraceno fueron biodegradados en un $16 \%, 13 \%$ y $9 \%$, respectivamente (Tabla 3). El resto de los PAH estudiados no fueron biodegradados, o su porcentaje de biodegradación fue menor a 9\%. Los suelos de la Patagonia poseen microorganismos de los géneros Pseudomonas, Alcaligenes, Rhodococcus, Sphingomonas y Mycobacterium [4, 5, 32] con capacidad de metabolizar PAH como fenantreno, pireno, benzoantreceno y benzopireno [21, 33, 34]. En el sistema de EBR estudiado se observó que fueron biodegradados los mismos PAH que en el sistema control, pero el porcentaje de biodegradación de cada uno fue superior. Por otro lado, la aplicación de corriente directa provocó la biodegradación de fenatreno, 1,3 metilfenantreno, criseno, 3 metilcriseno, 6 metilcriseno, benzo(b)fluorantreno y benzo(ghi)pireno, ya que en el sistema control no fueron utilizados como fuente de carbono por los microorganismos presentes (Tabla 3). El criseno es un PAH que está presente en los hidrocarburos extraídos en la zona, y es uno de los más resistentes a la biodegradación en sistemas adecuados para tal fin. La aplicación de EBR al suelo estudiado, provocó que este compuesto fuera biodegradado, ya que en el sistema control no se modificó su concentración, coincidiendo con lo observado por otros autores [35]. Esto pudo estar asociado a la presencia de un flujo electroosmótico provocado por la aplicación de corriente, capaz de removerlo desde el interior de los poros de las partículas del suelo, aumentando así su biodisponibilidad para ser biodegradado por los microorganismos del suelo [3]. Las diferencias observadas en los porcentajes de biodegradación de PAH totales en el cátodo, centro y ánodo, pueden deberse a que el flujo electroosmótico generado en el suelo no sea uniforme, siendo esto el resultado de que algunas secciones pueden presentar una presión en los poros variables, probablemente como resultado de la naturaleza compleja de los factores que controlan el flujo electroosmótico como ser el potencial zeta y el gradiente eléctrico [18]. 
Tabla 3. Concentración de los hidrocarburos poliaromáticos (PAH) determinados al inicio y al final de la experiencia. Los valores se encuentran expresados en mg. $\mathrm{kg}^{-1}$.

\begin{tabular}{lccccc}
\hline \multicolumn{1}{c}{ PAH } & Inicial & Control & Cátodo & Centro & Ánodo \\
\hline Naftaleno & 1.83 & 1.54 & 1.27 & 1.50 & 1.47 \\
1-Metilnaftaleno & 0.02 & 0.02 & 0.02 & 0.02 & 0.02 \\
1,6-Dimetilnaftaleno & 0.06 & 0.05 & 0.06 & 0.03 & 0.04 \\
Acenaftileno & 0.18 & 0.15 & 0.16 & 0.08 & 0.16 \\
Fenantreno & 0.05 & 0.06 & 0.00 & 0.05 & 0.00 \\
9-Metilfenantreno & 0.06 & 0.08 & 0.07 & 0.07 & 0.06 \\
1,7-Dimetilfenantreno & 0.65 & 0.51 & 0.00 & 0.06 & 0.08 \\
1,3-Metilfluoranteno & 0.51 & 0.50 & 0.32 & 0.35 & 0.38 \\
Reteno & 0.98 & 0.89 & 0.76 & 0.76 & 0.85 \\
Pireno & 0.86 & 0.75 & 0.66 & 0.76 & 0.71 \\
4-Metilpireno & 1.28 & 1.28 & 1.24 & 1.32 & 1.23 \\
1-Metilpireno & 0.54 & 0.55 & 0.54 & 0.52 & 0.55 \\
Criseno & 0.23 & 0.23 & 0.10 & 0.19 & 0.13 \\
3-Metilcriceno & 1.08 & 1.19 & 0.67 & 0.35 & 0.90 \\
6-Metilcriceno & 0.48 & 0.58 & 0.28 & 0.27 & 0.43 \\
Benzoantreceno & 3.85 & 3.51 & 2.98 & 3.26 & 2.97 \\
Benzo(b)fluorantreno & 0.90 & 0.84 & 0.74 & 0.91 & 0.81 \\
Dibenzo(a,h)antreceno & 0.37 & 0.37 & 0.35 & 0.34 & 0.35 \\
Benzo(ghi)pireno & 0.73 & 0.75 & 0.62 & 0.59 & 0.66 \\
PAH totales & 14.67 & 13.93 & 10.84 & 11.43 & 11.81 \\
\hline
\end{tabular}

\section{Conclusiones}

La electrobiorremediación es una técnica posible de ser aplicada en suelos no saturados de la Patagonia. Esta tecnología demostró buena capacidad de incrementar la biodegradación de hidrocarburos n-alcanos y en mayor medida, de los hidrocarburos poliaromáticos como fenatreno, 1,3 metilfenantreno, criseno, 3 metilcriseno, 6 metilcriseno, benzo(b)fluorantreno y benzo(ghi)pireno, que sin la aplicación de corriente directa, no fueron biodegradados por los microorganismos del suelo. La utilización de puentes salinos de fosfato de amonio, presentó problemas de liberación de amoníaco gaseoso, que si bien en los 60 días de estudio no representó un problema para el proceso de biodegradación, en sistemas de mayor duración pueden ocasionar inconveniente con el pH del suelo.

\section{Referencias}

1. J. Van Hamme, A. Singh, O. Ward, Microbiol. Mol. Biol. Rev. 67 (2003) 503-549. [10.1128/MMBR.67.4.503-549.2003]

2. $\quad$ G.N. Pucci, O.H. Pucci, Rev. Argent. Microb. 35 (2003) 62-68.

3. A.R. Johnsen, L.Y. Wick, H. Harms, Environ. Pollut. 133 (2005) 71-84. [10.1016/j.envpol.2004.04.015]

4. A.J. Acuña, O.H. Pucci, G.N. Pucci, Ecosistemas 17 (2008) 85-93.

5. S.R. Peressutti, H.M. Alvarez, O.H. Pucci, Int. Biodeterior. Biodegrad. 52 (2003) 21-30. [10.1016/S0964-8305(02)00102-6] 
6. M. Maila, T. Cloete, Rev. Environ. Sci. Biotechnol. 3 (2004) 349-360. [10.1007/s11157-004-6653-z]

7. D.A. De la Rosa-Pérez, M.M. Teututi-León, M.E. Ramírez-Islas, Rev. Int. Contam. Ambient. 23 (2007) 129-138.

8. L.Y. Wick, L. Shi, H. Harms, Electrochim. Acta 52 (2006) 3441-3448. [10.1016/j.electacta.2006.03.117]

9. S. Laursen, Can. Geotec. J. 43 (1997) 664-671. [10.1139/cgj-34-5-664]

10. Y. Acar, A. Alshawabkeh, Environ. Sci. Technol. 27 (1993) 2638-2647. [10.1021/es00049a002]

11. Y.B. Acar, R.J. Gale, A.N. Alshawabkeh, R.E. Marks, S. Puppala, M. Bricka, R. Parker, J. Hazard. Mater. 40 (1995) 117-137. [10.1016/03043894(94)00066-P]

12. S.O. Kim, K.W. Kim, J. Hazard. Mater. 85 (2001) 195-211. [10.1016/S0304-3894(01)00211-4]

13. K.R. Reddy, S. Chinthamreddy, Waste Manage. 19 (1999) 269-282. [10.1016/S0956-053X(99)00085-9]

14. P. Suer, K. Gitye, B. Allard, Environ. Sci. Technol. 37 (2003) 177-181. [10.1021/es010226h]

15. G. Maini, A. Sharman, in Bioremediation of Metal and Inorganic Compounds, A. Lesson, B. Alleman, Eds., Battelle press, USA, 1999, p. 127.

16. R. Marks, Y. Acar, R. Gale, in Remediation of Hazarous Waste Contaminated Soil, D. Wise, D. Trantolo, Eds., Marcel Dekker, New York, 1994, p. 405.

17. J. Virkutyte, M. Sillanpää, P. Latostenmaa, Sci. Total Environ. 289 (2002) 97-121. [10.1016/S0048-9697(01)01027-0]

18. J.L. Niqui-Arrollo, M. Bueno-Montes, R. Posada-Vaquero, J.J. OrtegaCalvo, Environ. Pollut. 142 (2006) 326-332. [10.1016/j.envpol.2005.10.007]

19. Garcia Trejo, Experimentos en Microbiología del Suelo, Compañía Editorial Continental S.A., México, 1981, p. 14.

20. Environmental Pollution Agency. Environmental Protection Agency (2003) Method 8015D: Nonhalogenated Organics Using GC/FID.

21. L.Y. Wick, P.A. Mattle, P. Wattiau, H. Harms, Environ. Sci. Technol. 38 (2004) 4596-4602. [10.1021/es0354420]

22. D.J. Reasoner, E.E. Geldereich, Appl. Environ. Microbiol. 49 (1985) 1-7.

23. V. Riis, S. Kleinsteuber, W. Babel, Can. J. Microbiol. 49 (2003) 713-721. [10.1139/w03-083]

24. S. Kleinsteuber, V. Riis, I. Fetzer, H. Harms, S. Muller, Appl. Environ. Microbiol. 72 (2006) 3531-3542. [10.1128/AEM.72.5.3531-3542.2006]

25. C.A.B. Schmidt, M.C. Barbosa, M.S.S. Almeida, J. Hazard. Mater. 143 (2007) 655-661. [10.1016/j.jhazmat.2007.01.009]

26. E.R. Lindgren, E.D. Mattson, M.W. Kosak, Am. Chem. Soc. Symp. S. 554 (1994) 33-50. [10.1021/bk-1994-0554.ch003]

27. A.P. Shapiro, R.F. Probstein, Environ. Sci. Technol. 27 (1993) 283-291. [10.1021/es00039a007] 
28. M.J. Harbottle, G. Lear, G.C. Sills, I.P. Thompson, J. Environ. Manage. 20 (2009) 1893-1900. [10.1016/j.jenvman.2008.12.012]

29. S. Suni, M. Romantshuk, FEMS Microbiol. Ecol. 49 (2004) 51-57. [10.1016/j.femsec.2004.01.016]

30. M.F. DeFlaun, C.W. Condee, J. Hazard. Mater. 55 (1997) 263-277. [10.1016/S0304-3894(97)00023-X]

31. M.E. Bayer, J.L. Sloyer, J. Gen. Microbiol. 136 (1990) 867-874. [10.1099/00221287-136-5-867]

32. G.N. Pucci, O.H. Pucci, Acta Biol. Colom. 11 (2006) 19-31

33. R.F. Wang, W.W. Cao, C.E. Cerniglia, FEMS Microbiol. Lett. 130 (1995) 75-80. [10.1111/j.1574-6968.1995.tb07701.x]

34. J.D. Moody, J.P. Freeman, P.P. Fu, C.E. Cerniglia, Appl. Environ. Microbiol. 70 (2004) 340-345. [10.1128/AEM.70.1.340-345.2004]

35. L. Coror-Ramírez, R. Iturbe-Argüelles, Ing. Invest. Tecnol. 6 (2004) 119126. 